\title{
Use of reinforced earth to improve the stability of railway sleepers
}

\section{Utilisation de la terre armée pour l'amélioration de la stabilité des traverses ferroviaires}

\author{
Souad ATOUB ${ }^{1}$, Riad BENZAID ${ }^{2}$, Mustapha TEKKOUK ${ }^{3}$ \\ 1,2,3 Laboratoire de Génie Géologique, Université Mohammed Seddik Benyahia, Jijel, Algérie.
}

\begin{abstract}
Soil reinforcement with inclusion has been used since the dawn of time, however, decisive progress in this area dates back only a few decades, When the techniques of soil reinforcement are passed to an industrial development with metallic inclusions then geosynthetic and for applications as well in embankments as in cuttings. Reinforced earth is one of the most used methods for soil strengthening. It is a relatively recent technique whose principle consists in associating with a cohesionless soil tensile reinforcements, which thus give the material an anisotropic cohesion in the direction of the reinforcements. Among the areas where the reinforced earth is currently experiencing a remarkable expansion, railway infrastructure, traditional means for the transport of passengers and goods, and which has never ceased to be the subject of technical progress. This progress based for a long time on the empiricism, allowed the progressive increase, as well speeds as tonnage by axle. However, the railway remains a privileged place of deformation under the effect of the repetitive application of loads. These deformations are largely related to the problem of ballast settlement, a problem whose causes are diverse and that we will try to study in the laboratory on a physical model in real size. The present work consists in experimentally analyzing the load-settlement relationships that exist during the introduction of stainless steel reinforcement into an unstable rail ballast under static vertical loading and to establish the conditions that lead to the reduction of recorded settlements in order to improve the stability of railway sleepers.
\end{abstract}

Résumé. Le renforcement des sols à l'aide d'inclusion a été utilisé depuis l'aube du temps cependant, les progrès décisifs dans ce domaine ne datent que de quelques dizaines d'années, lorsque les techniques de renforcement des sols sont passées à un développement industriel avec des inclusions métalliques puis géosynthètiques et à des applications aussi bien en remblais qu'en déblais. La terre armée est l'un des procédés les plus utilisés pour le renforcement des sols. C'est une technique relativement récente dont le principe consiste à associer à un sol pulvérulent des armatures résistantes à la traction, qui confèrent ainsi au matériau une cohésion anisotrope, dans la direction des armatures. Parmi les domaines où la terre armée connaît actuellement une remarquable expansion, les infrastructures des chemins de fer, moyen traditionnel pour le transport des voyageurs et des marchandises, et qui n'a jamais cessé d'être l'objet de progrès techniques. Ce progrès basé longtemps sur l'empirisme, a permis l'augmentation progressive, tant des vitesses que du tonnage par essieu. Cependant, la voie ferrée reste un siège privilégié de déformations sous l'effet de l'application répétitive des charges. Ces déformations sont liées en grande partie au problème de tassement du ballast, un problème dont les causes sont diverses et qu'on va essayer de l'étudier en laboratoire sur un modèle physique en grandeur réelle. Le présent travail consiste à analyser par voie expérimentale, les relations charge-tassement qui existent lors d'une introduction des armatures inoxydable dans un ballast ferroviaire instable sous chargement vertical statique et d'établir les conditions qui mènent à la réduction des tassements enregistrés afin d'améliorer la stabilité des traverses ferroviaires. 


\section{Introduction}

La voie ferrée connaît actuellement une profonde évolution et tend à s'adapter aux besoins de notre époque. Des progrès considérables ont été réalisés ces dernières années tant dans le domaine des performances pures que dans celui de l'exploitation courante. Cela s'est entre autres traduit par l'utilisation de plus en plus fréquente des grandes vitesses, une augmentation du confort des voyageurs, du tonnage par essieu, et par une diminution des dépenses d'entretien [1,2].

Cependant, sous l'effet de l'application répétitive de la charge, la voie ferrée reste un siège continu de déformations. Ces déformations ou dégradations de la voie sont en grande partie liées aux problèmes de tassement du ballast [3].

Le maintien de la géométrie de la voie ferrée est directement lié au comportement du ballast. Ce dernier, a pour rôle essentiel de transmettre au sol la charge qu'exerce le rail sur le blochet en veillant à s'opposer aux mouvements longitudinaux et transversaux des traverses $[4,5]$.

\section{Principe général du comportement du $D$ ballast armé}

Il est admis que sous l'effet d'une charge verticale, l'armature introduite dans le ballast ferroviaire diminue les déformations latérales et verticales du milieu pulvérulent. Ainsi admise, cette hypothèse est vérifiable au cas où les forces de frottement entre les grains du milieu pulvérulent et la surface de l'armature seraient suffisantes. Un tel cas limitera le glissement latéral, par conséquent, les déformations verticales du ballast sont à leur tour atténuées et le tassement du sol minimisé.

En empêchant le glissement latéral des grains du milieu pulvérulent, l'armature (sous forme de treillis soudé) transmet les efforts de traction et assure une cohésion locale de la couche de ballast [6, 7]. Ce phénomène correspond à une augmentation locale de l'angle de frottement interne du sol.

L'interaction sol-armature développée est de deux types $[6,7]$ :

- Un frottement latéral entre les éléments orientés dans le sens de la traction ;

- Une résistance en butée le long des éléments transversaux.

Lorsque le sol remplit les ouvertures des treillis soudés, une portion de la résistance du mécanisme est désorientée aux fils d'armatures transversales des treillis. Les contraintes dans les fils d'armatures transversales sont transférées aux fils longitudinaux qui résiste aux contraintes imposées, ces contraintes vont être transférées à travers les nœuds (d'où on remarque leur importance). C'est l'effet de "raquette" où le système de renforcement bidimensionnel répartit les charges sur le sol, tout comme les raquettes soutiennent le poids d'un homme sur la neige (Figure 1).

\section{Dimensionnement des renforcements}

La procédure de dimensionnement applique depuis longtemps le concept d'équilibre plastique de Rankine qui utilise les conditions des pressions des terres actives pour les charges permanentes et le concept de Boussinesq pour les charges de service.

Notons que la force admissible du treillis soudé considérée à la moindre apparition des dommages ou glissements doit être inclue dans le dimensionnement.

La valeur de la force de traction maximale dans le treillis soudé $\left(T_{\max }\right)$ est calculée en fonction de sa déformation maximale $\left(\varepsilon_{\max }\right)$ par la formule suivante :

$T_{\text {max }}=\left(T_{a} / \varepsilon_{a}\right) \varepsilon_{\text {max }}$

Avec $\left(T_{a} / \varepsilon_{a}\right)$ le module de traction du treillis soudé.

L'équilibre interne du massif de sol est satisfait lorsque la composante horizontale de la poussée latérale totale est égale à la somme des tractions dans tous les fils de renforcements :

$\sum T_{\max }=0,5 K_{a} \cdot \gamma \cdot H^{2}+K_{q} \cdot q \cdot H$

$K_{a}:$ le coefficient des poussées des terres actives.

$K_{q}$ : le coefficient de transmission des surcharges.

$H$ : l'épaisseur du ballast au-dessus du renforcement.

$q$ : les surcharges ferroviaires.

En connaissant la résistance maximale fournie par le treillis soudé ( $R T_{\max }$ unitaire), on peut déduire le choix et le nombre de treillis adéquat par mètre linéaire ainsi que la détermination des déformations maximales $\left(\varepsilon_{\max }\right)$.

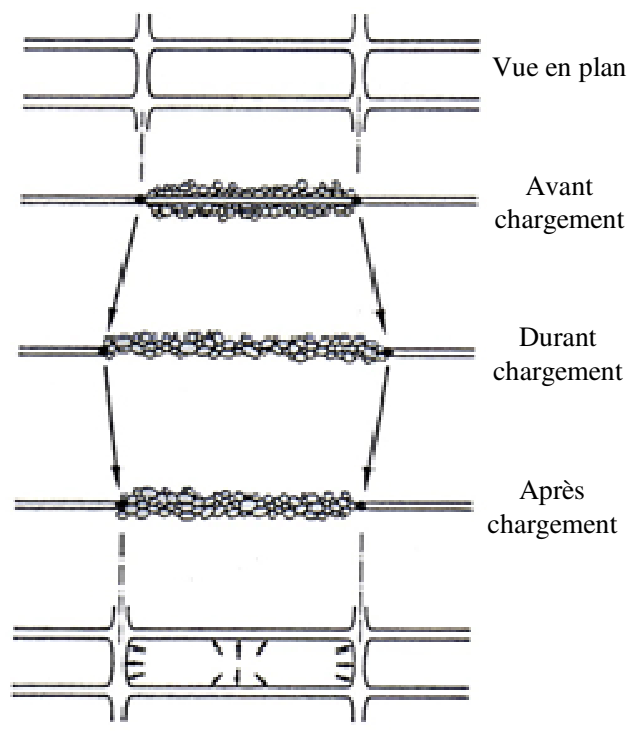

Fig. 1. Mécanisme de mise en tension des treillis soudés.

\section{Objectif des essais}

On se propose d'analyser par voie expérimentale les relations qui apparaissent lors de l'introduction d'armatures de renforcement dans un ballast ferroviaire instable et, d'établir les conditions qui permettraient de réduire le tassement de ce dernier. Notre étude se limite 
aux déformations du ballast dans les sections de voies ferroviaires d'accès au garage ou d'entretien et aux autres tronçons où des déformations sont observées au niveau de la couche du ballast ferroviaire.

Par l'expérience menée au laboratoire, nous essayerons de connaître :

- L'effet du renforcement sur le tassement de la couche de ballast et par conséquent, l'influence sur la stabilité des traverses ferroviaires.

- L'effet du changement de la position en profondeur des armatures de renforcement sur la valeur du tassement.

\section{Mode d'exécution de l'expérience}

Le dispositif de laboratoire ayant servi à la réalisation de nos essais de simulation est représenté en Figure 2.
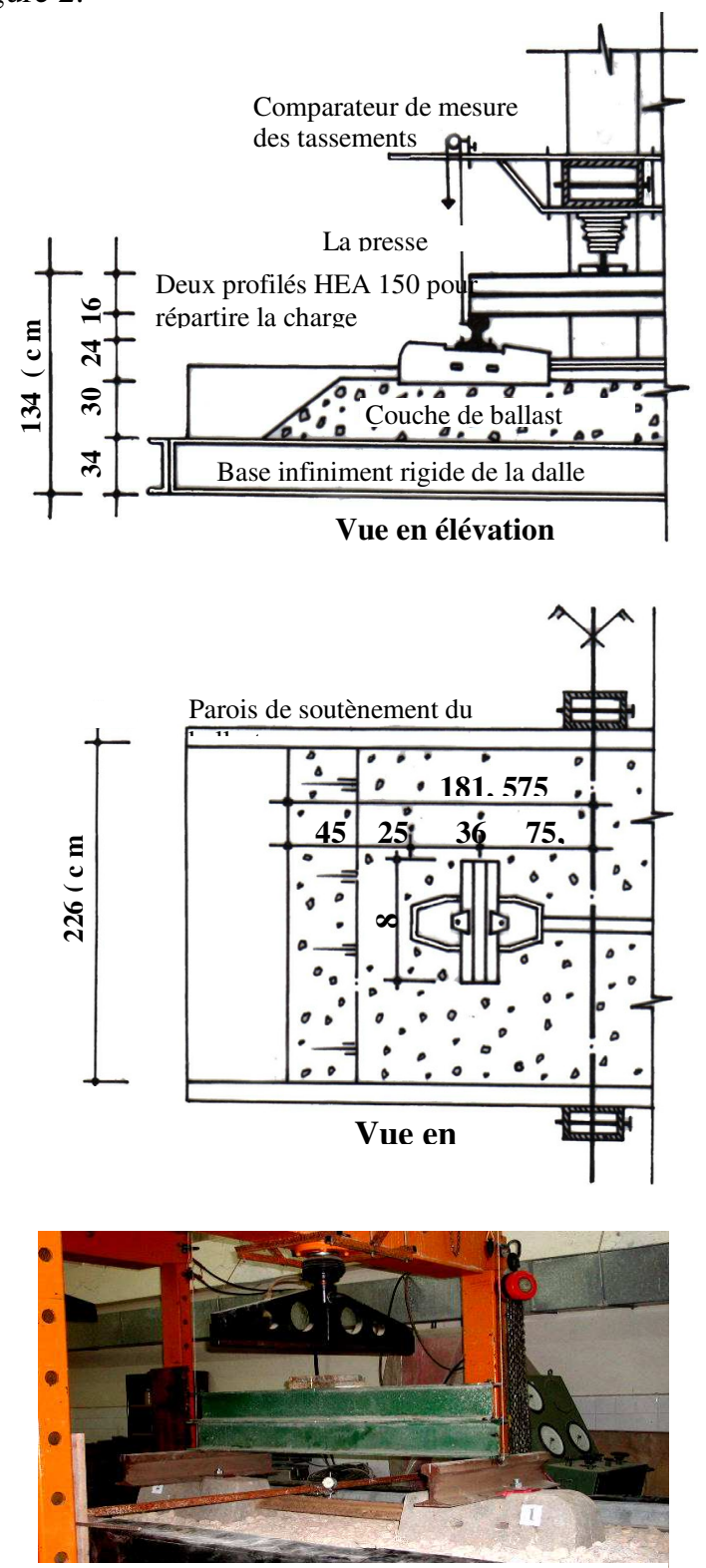

Vue générale du dispositif d'essai

Fig. 2. Banc d'essai
Composé d'une base infiniment rigide, le banc d'essai supporte la couche de ballast étudiée. Cette couche de ballast d'épaisseur $30 \mathrm{~cm}$ et de dimensions en plan de $(363,15 \mathrm{~cm} \times 226 \mathrm{~cm})$ est chargée d'une traverse ferroviaire en béton armé de type SL. Les deux blochets en bêton armé ont chacun les dimensions suivantes : $\mathrm{B}=$ $29 \mathrm{~cm}, \mathrm{~L}=68 \mathrm{~cm}$ et $\mathrm{h}=24 \mathrm{~cm} \mathrm{[4].}$

L'armature de renforcement utilisée est un treillis soudé constitué de fil de fer cylindrique de diamètre 4 $\mathrm{mm}$, il se présente en mailles carrées de $(15 \mathrm{~cm}$ x $15 \mathrm{~cm})$, disposé horizontalement. Ce type d'armature a été choisi sur la base des remarques suivantes: il doit être suffisamment résistant à la traction, peu extensible (à cause de l'assemblage des fils au nœud), durable (acier inoxydable).

Notons que la forme en mailles carrées du treillis soudé ne divise pas le sol en deux couches séparées, son seul but est d'augmenter localement l'effet de l'angle de frottement interne du sol et de résister aux forces de tractions latérales.

La couche de ballast de granulométrie $25 / 50 \mathrm{~mm}$, est soumise à l'aide d'une presse hydraulique à une sollicitation maximale de deux forces verticales statiques de 10 tonnes chacune distantes de $151,15 \mathrm{~cm}$ (ce qui représente l'entre axe de la traverse SL). Le pas de chargement appliqué est de 1 tonne jusqu'à une valeur de 10 tonnes par blochet, ce qui correspond à une charge totale de 20 tonnes par essieu (catégorie $\mathrm{C}$ des voies classées par l'Union Internationale des Chemins de fer en ce qui concerne les efforts supportés par la voie ferrée).

Un comparateur de déplacement placé au-dessus de chaque blochet de la traverse ferroviaire chargée mesure les tassements au cours des essais (voir Figure 1).

Deux séries d'essais ont été réalisés pour chaque disposition du programme expérimental (ballast de référence et ballast renforcé). Après chaque série d'essai, le ballast est remis à son état initial.

\section{Programme d'essais}

Essai $n^{\circ} 1$ :

C'est l'essai de référence (Figure 3), la traverse ferroviaire est placée sur une couche de ballast ordinaire.

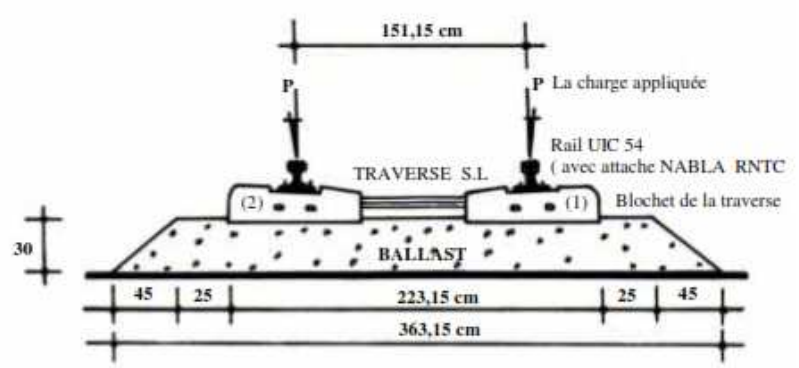

Fig. 3. Essai de référence

Essai $n^{\circ} 2$ :

Essai renforcé 1 (Figure 4), la traverse ferroviaire est placée sur une couche de ballast renforcé avec un treillis soudé introduit à mi-hauteur. 


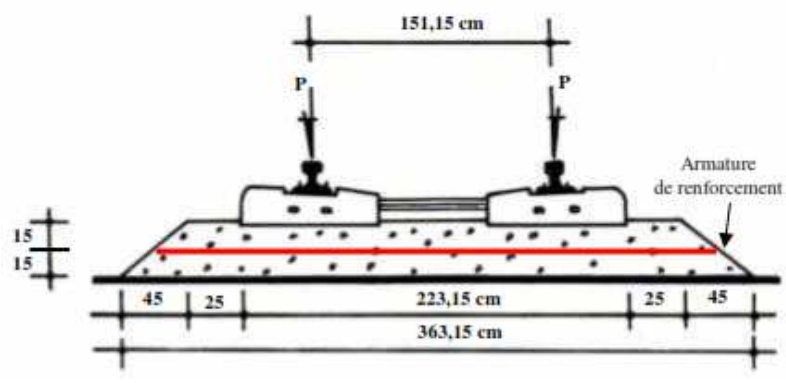

Fig. 4. Essai renforcé (1)

Essai $n^{\circ} 3$ :

Essai renforcé 2 (Figure 5), la traverse ferroviaire est placée sur une couche de ballast renforcé avec un treillis soudé introduit à $7,5 \mathrm{~cm}$ de la surface.

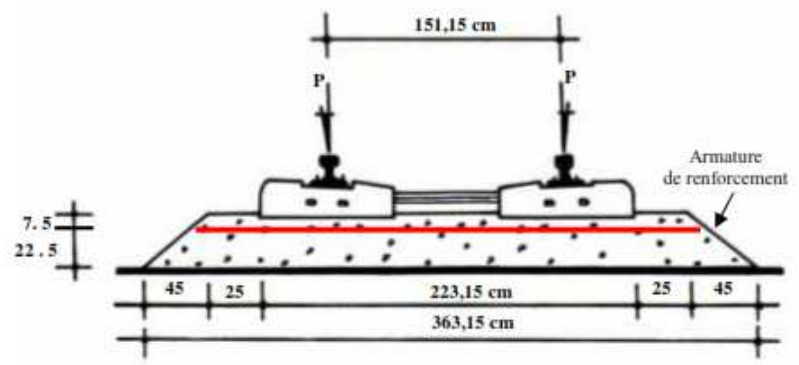

Fig. 5. Essai renforcé (2)

\section{Etude et analyse des résultats expérimentaux}

\subsection{Tassements de la couche du ballast}

La couche de ballast est chargée d'une traverse ferroviaire soumise à une sollicitation de deux forces verticales symétriques appliquées sur les rails au niveau des blochets. Le pas de chargement simultanée est de 1 tonne et la charge maximale exercée par blochet est de 10 tonnes.

\subsubsection{Cas du ballast de référence}

La courbe de la Figure 6 donne l'évolution du tassement le long de l'expérience. Dans ce cas de figure la valeur du tassement moyen est de l'ordre de 2,69 cm.

\subsubsection{Cas du ballast renforcé}

Renforcé avec un treillis soudé à mi-hauteur et, à 7,5 $\mathrm{cm}$ de sa surface, la couche de ballast tasse moins (Figure 6). Le tassement est nettement réduit par rapport à l'essai de référence, avec des valeurs moyennes de l'ordre de $1,255 \mathrm{~cm}$ et $1,074 \mathrm{~cm}$ respectivement.

Les différences entre les tassements de la traverse sur ballast renforcé et non renforcé sont considérables. Les tassements de la traverse surmontant le ballast renforcé à mi-hauteur, sont deux fois plus faibles que ceux du ballast de référence. En effet, le tassement moyen enregistré pour les deux essais de référence a été réduit d'environ $53 \%$ après le renforcement de la couche de ballast avec un treillis soudé disposé à sa mi-hauteur.

$\mathrm{La}$ réduction $\mathrm{du}$ tassement est nettement plus marquée quand l'armature de renforcement est introduite à $7,5 \mathrm{~cm}$ de la surface du ballast. En effet, le tassement moyen obtenu sur le ballast de référence est réduit de 60 $\%$.

Les réductions des tassement ainsi obtenues sont dues aux frottements développés entre les grains du ballast et la surface des armatures de renforcement. Les mailles du treillis soudé opposent une butée qui empêche le glissement des grains "effet de raquette". L'interaction sol-armature contribue à améliorer l'adhérence des deux composants du ballast renforcé. Sous ces conditions (frottement mobilisé est adhérence améliorée), la couche de ballast aura un glissement latéral limité et par conséquent des déformations verticales atténuées. Le glissement latéral des grains ainsi empêché, l'armature aidera à transmettre les efforts de traction tout en assurant la cohésion locale de la couche du ballast.
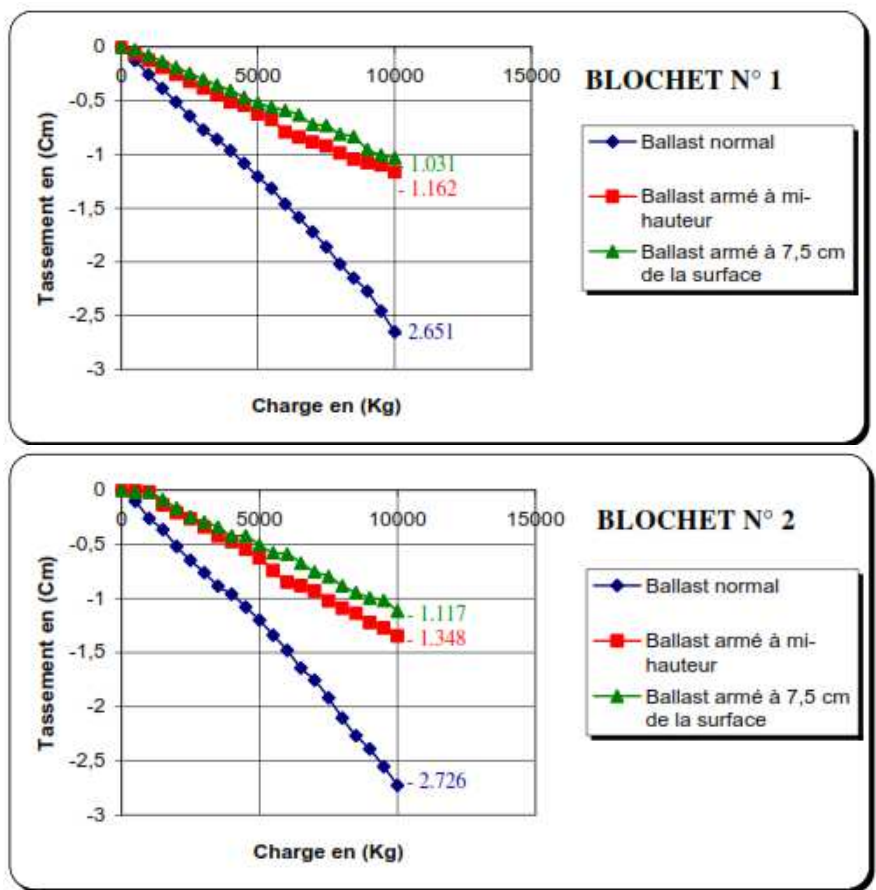

Fig. 6. Tassement sous le blochet $n^{\circ} 1$ et $n^{\circ} 2$ de la traverse chargée sur du ballast armé et non armé

Notons que l'intérêt de l'utilisation des treillis soudé comme armatures de renforcement réside dans le fait que la couche renforcée garde son unicité, elle n'est nullement stratifiée ou divisée en deux une fois l'armature introduite en son sein. Cette même armature participe à augmenter l'effet de l'angle de frottement interne du ballast par la transmission des efforts de traction ce qui induit la diminution des déformations latérales dues aux charges verticales appliquées. 


\subsection{Influence de la disposition des armatures de renforcement au niveau du ballast}

Le changement de la disposition en profondeur des armatures de renforcement à plus ou moins influencé les valeurs des tassements de la couche du ballast (Figure 6). En effet, placée à $7,5 \mathrm{~cm}$ de la surface, l'armature de renforcement a contribué à réduire d'avantage le tassement par rapport à la disposition à mi-hauteur.

Ainsi donc, le tassement moyen enregistré à mihauteur est réduit de $14 \%$ une fois que les armatures été placées plus haut, soit à $7,5 \mathrm{~cm}$ de la surface du ballast. Cela s'explique par le fait que le coefficient de frottement apparent entre le sol et les armatures diminue en fonction de la profondeur [8].

D'autre part, le sol sera moins dilatant si la pression de confinement est plus élevée. Ceci explique pourquoi l'effet favorable de la dilatance sur le coefficient de frottement apparent diminue lorsque la contrainte verticale moyenne $(\gamma . \mathrm{H})$ exercée par le ballast sur les armatures augmente. Le coefficient de frottement apparent sera diminué et les forces de frottement solarmature réduites.

Tous ces éléments expliquent parfaitement les conditions de l'amélioration du renforcement du sol étudié.

Par le biais de ces résultats, nous confirmons que le ballast renforcé avec des treillis soudés a gardé le même principe de comportement que celui des massifs en terre armée.

\section{Conclusions}

Le travail présenté est une étude expérimentale sur un modèle physique en grandeur réelle, il consiste à introduire des treillis soudés dans un ballast ferroviaire sollicité par un chargement vertical dans le but de minimiser les tassements de ce dernier.

A travers cette recherche, nous pouvons tirer les conclusions suivantes :

- Les résultats obtenus montrent que le ballast renforcé est très utile dans les superstructures ferroviaires instables.

- L'utilisation du treillis soudé peut améliorer la stabilité de la superstructure des tronçons de voies où le ballast manifeste d'une façon permanente des déformations importantes.

- Les efforts de tractions au sein du ballast renforcé sont repris par la mobilisation de frottement entre le milieu pulvérulent et l'armature, ce qui a pour effet de limiter les déformations horizontales et verticales de la couche considérée.

- Le tassement du ballast renforcé est d'environ $50 \%$ plus faible que celui du ballast de référence.

- L'utilisation des treillis soudés permet d'obtenir un renforcement bidimensionnel. Ce qui favorise d'une part, la répartition des contraintes dans la couche renforcée et d'autre part, permettra d'avoir un frottement sol-armature dans les deux directions du renforcement "effet de raquette".
En perspectives, le frottement sol-armature peut être amélioré par l'augmentation de la surface de contact. Pour cela l'utilisation de lanières métalliques plus larges est conseillée. D'autre part, ces essais menés en laboratoire peuvent servir de base à d'autres études sur des ouvrages réels à caractère expérimental.

Le système de renforcement proposé dans ce travail peut trouver application dans les cas pratiques suivants :

(a) Les passages à niveau entre les routes et les chemins de fer, où la différence de rigidité entre les deux voies est à l'origine des déformations souvent rencontrées.

(b) La décompression des dalles de transitions à l'entrée des ouvrages d'art ferroviaires.

(c) Les sections des voies ferroviaires construites sur sol compressible.

(d) Les sections des voies ferroviaires servant comme voies de garage ou d'entretien.

(e) Les voies anciennes.

\section{Références bibliographiques}

[1] Renforcement des Sols : Expérimentation En vraie grandeur des Années 80', Symposium international sur le renforcement des sols, Paris 18-19 novembre 1993, presse de l'ENPC (1993).

[2] V. Profillidis, P. Humbert. Etude en élastoplasticité par la méthode des éléments finis du comportement de la voie ferrée et de sa fondation - bull. LPC, 141, réf. 3051 (1986)

[3] A. Al-Shaer. Permanent settlement analysis of ballasted railway tracks - Dynamical approach. Engi Sci. Ecole des Ponts, ParisTech, (2005).

[4] SNTF. Entretien de la voie: Le Ballast. Document technique destiné au personnel d'encadrement de la SNTF (Algérie), Réalisé par: SOFRERAILSOFRETU- France (2000).

[5] S. Malab Mankour, A. Mansouri, A. Benaissa. Comportement du ballast sous chargement vertical pour différentes structures de l'assise ferroviaire. Séminaire national de génie civil, Sidi Bel Abbés, Algérie. 15 -16 mai (2001).

[6] R. Benzaid. Stabilité des traverses ferroviaires sur un ballast armé sous chargement statique sur un support rigide, étude expérimentale - Mémoire de magister, Université badji-mokhtar, Annaba, Algérie (2002).

[7] M. Tekkouk, R. Benzaid. La terre armée, une solution d'amélioration des fondations pour remblais en terrain meuble. XXIX ${ }^{\text {eme }}$ Rencontres Universitaires de Génie Civil. Tlemcen, 29 au 31 Mai (2011).

[8] F. Schlosser, M. Bastick. Reinforced earth, In Foundation Engineering Hand Book, second edition, edited by: Hsai-Yang Fang. Chapman and hall, New York, pp. 778-795 (1991). 